\title{
Vital Signs: Deaths Among Persons with Diagnosed HIV Infection, United States, 2010-2018
}

\begin{abstract}
Karin A. Bosh, $\mathrm{PhD}^{1}$; Anna Satcher Johnson, $\mathrm{MPH}^{1}$; Angela L. Hernandez, MD ${ }^{1}$; Joseph Prejean, $\mathrm{PhD}^{1}$; Jocelyn Taylor, MPH ${ }^{1}$; Rachel Wingard, $\mathrm{MA}^{1}$; Linda A. Valleroy, $\mathrm{PhD}^{1}$; H. Irene Hall, $\mathrm{PhD}^{1}$
\end{abstract}

\section{Abstract}

Background. Life expectancy for persons with human immunodeficiency virus (HIV) infection who receive recommended treatment can approach that of the general population, yet HIV remains among the 10 leading causes of death among certain populations. Using surveillance data, CDC assessed progress toward reducing deaths among persons with diagnosed HIV (PWDH).

Methods. CDC analyzed National HIV Surveillance System data for persons aged $\geq 13$ years to determine age-adjusted death rates per 1,000 PWDH during 2010-2018. Using the International Classification of Diseases, Tenth Revision, deaths with a nonmissing underlying cause were classified as HIV-related or non-HIV-related. Temporal changes in total deaths during 2010-2018 and deaths by cause during 2010-2017 (2018 excluded because of delays in reporting), by demographic characteristics, transmission category, and U.S. Census region of residence at time of death were calculated.

Results. During 2010-2018, rates of death decreased by 36.6\% overall (from 19.4 to 12.3 per 1,000 PWDH). During 2010-2017, HIV-related death rates decreased 48.4\% (from 9.1 to 4.7), whereas non-HIV-related death rates decreased 8.6\% (from 9.3 to 8.5). Rates of HIV-related deaths during 2017 were highest by race/ethnicity among persons of multiple races (7.0) and Black/African American persons (5.6), followed by White persons (3.9) and Hispanic/Latino persons (3.9). The HIV-related death rate was highest in the South (6.0) and lowest in the Northeast (3.2).

Conclusion. Early diagnosis, prompt treatment, and maintaining access to high-quality care and treatment have been successful in reducing HIV-related deaths and remain necessary for continuing reductions in HIV-related deaths.

\section{Introduction}

Persons with human immunodeficiency virus (HIV) infection require lifelong treatment to reduce HIV-related morbidity and mortality; advances in HIV treatment have resulted in a life expectancy that approaches that of the general population $(1,2)$. Deaths attributable to HIV infection are preventable, yet during 2017, HIV was still among the 10 leading causes of death among certain population groups (3).

The National HIV Surveillance System (NHSS) is the primary source of population-based information about HIV in the

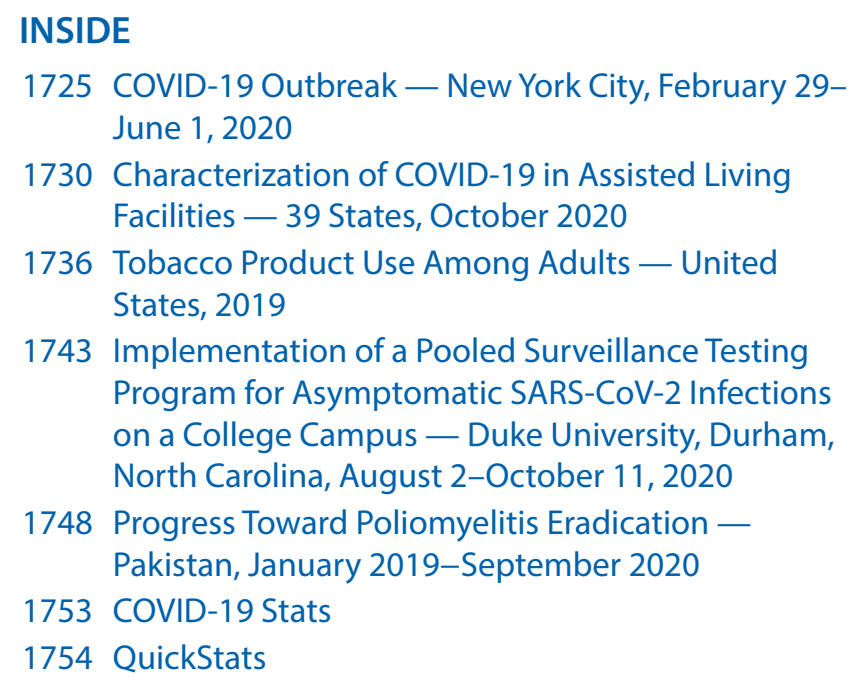

1725 COVID-19 Outbreak - New York City, February 29June 1, 2020

1730 Characterization of COVID-19 in Assisted Living Facilities - 39 States, October 2020

1736 Tobacco Product Use Among Adults — United States, 2019

1743 Implementation of a Pooled Surveillance Testing Program for Asymptomatic SARS-CoV-2 Infections on a College Campus - Duke University, Durham, North Carolina, August 2-October 11, 2020

1748 Progress Toward Poliomyelitis Eradication Pakistan, January 2019-September 2020

1753 COVID-19 Stats

1754 QuickStats

Continuing Education examination available at https://www.cdc.gov/mmwr/mmwr_continuingEducation.html

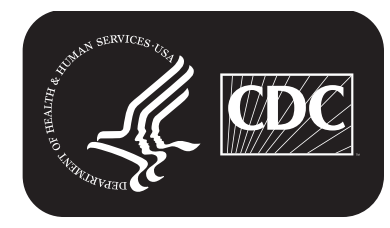


United States (4). A previous analysis demonstrated that, during 1990-2011, deaths among persons with stage 3 HIV infection (acquired immunodeficiency syndrome [AIDS]) decreased, with larger decreases in HIV-attributable deaths (-89\%) than in nonHIV-attributable deaths (-57\%) (5). On the basis of increasing evidence of the benefits of antiretroviral therapy both for persons with HIV and for preventing secondary transmission, treatment guidelines were updated in 2012 to recommend antiretroviral therapy for all persons with HIV (6). A national target for reducing the death rate among persons with diagnosed HIV (PWDH) by $\geq 33 \%$ during 2010-2020 was established to encourage progress toward improving health outcomes among PWDH (7). Using NHSS data, CDC assessed such progress, with an emphasis on HIV-related deaths, at the national and state levels.

\section{Methods}

CDC analyzed NHSS data reported through December 2019 regarding deaths during 2010-2018 among persons aged $\geq 13$ years with diagnosed HIV infection. Using the International Classification of Diseases, Tenth Revision (ICD-10) codes associated with the underlying cause, deaths were classified as HIVrelated or non-HIV-related.* Annual deaths (2010-2018) and

\footnotetext{
*HIV-related: deaths with an ICD-10 code of B20-B24, 098.7, or R75 for the underlying cause; non-HIV-related: all other deaths with a nonmissing ICD-10 code for the underlying cause.
}

deaths by cause (2010-2017 because of delays in reporting) were assessed by demographic characteristics, transmission category, and U.S. region of residence at time of death. Nationallevel results include persons with a residence at time of death in the 50 states or the District of Columbia; jurisdiction-level results also include persons with a residence at time of death in Puerto Rico.

Age-adjusted rates per 1,000 PWDH were calculated using the U.S. 2000 standard population. For HIV-related deaths, $\mathrm{CDC}$ calculated an absolute and a relative disparity measure for race/ethnicity and assessed change from 2010 to $2017 . \dagger, \$$ For all measures, only stable rates (calculated on the basis of $\geq 12$ deaths) and rates by cause of death for groups among whom $\geq 85 \%$ of deaths had a known cause (i.e., complete cause of death reporting) were assessed for temporal changes and for differences among groups.

\footnotetext{
$\dagger$ Absolute rate difference disparity measure: Difference between age-adjusted rate per 1,000 PWDH among selected race/ethnicity and White persons (population with lowest rate during 2010 among those with rates where $\geq 85 \%$ of deaths had a known cause). Change in the absolute rate difference disparity measure during 2010-2017 was calculated as ([absolute disparity measure in 2017 - absolute disparity measure in 2010]/absolute disparity measure in 2010) x 100 .

$\mathbb{S}$ Relative rate ratio disparity measure: Ratio of age-adjusted rates per 1,000 PWDH for selected race/ethnicity, compared with White persons (population with lowest rate during 2010 among those with rates where $\geq 85 \%$ of deaths had a known cause). Change in the relative rate ratio disparity measure during 2010-2017 was calculated as ([relative disparity measure in 2017 _ relative disparity measure in 2010]/relative disparity measure in 2010) x 100 .
}

The MMWR series of publications is published by the Center for Surveillance, Epidemiology, and Laboratory Services, Centers for Disease Control and Prevention (CDC), U.S. Department of Health and Human Services, Atlanta, GA 30329-4027.

Suggested citation: [Author names; first three, then et al., if more than six.] [Report title]. MMWR Morb Mortal Wkly Rep 2020;69:[inclusive page numbers].

\author{
Centers for Disease Control and Prevention \\ Robert R. Redfield, MD, Director \\ Anne Schuchat, MD, Principal Deputy Director \\ Ileana Arias, PhD, Acting Deputy Director for Public Health Science and Surveillance \\ Rebecca Bunnell, PhD, MEd, Director, Office of Science \\ Jennifer Layden, MD, PhD, Deputy Director, Office of Science \\ Michael F. Iademarco, MD, MPH, Director, Center for Surveillance, Epidemiology, and Laboratory Services
}

Charlotte K. Kent, PhD, MPH, Editor in Chief Jacqueline Gindler, MD, Editor

Paul Z. Siegel, MD, MPH, Guest Associate Editor

Mary Dott, MD, MPH, Online Editor

Terisa F. Rutledge, Managing Editor

Douglas W. Weatherwax, Lead Technical Writer-Editor

Glenn Damon, Soumya Dunworth, PhD,

Teresa M. Hood, MS, Donald G. Meadows, MA, Technical Writer-Editors

Matthew L. Boulton, MD, MPH

Carolyn Brooks, $\mathrm{ScD}$, MA

Jay C. Butler, MD

Virginia A. Caine, MD

Jonathan E. Fielding, MD, MPH, MBA

David W. Fleming, MD

MMWR Editorial and Production Staff (Weekly)

Martha F. Boyd, Lead Visual Information Specialist Alexander J. Gottardy, Maureen A. Leahy,

Julia C. Martinroe, Stephen R. Spriggs, Tong Yang, Visual Information Specialists

Quang M. Doan, MBA, Phyllis H. King,

Terraye M. Starr, Moua Yang,

Information Technology Specialists

MMWR Editorial Board

Timothy F. Jones, MD, Chairman

Kate Galatas, MPH

William E. Halperin, MD, DrPH, MPH

Jewel Mullen, MD, MPH, MPA

Jeff Niederdeppe, PhD

Celeste Philip, MD, MPH

Patricia Quinlisk, MD, MPH
Ian Branam, MA, Acting Lead Health Communication Specialist Shelton Bartley, MPH, Lowery Johnson, Jacqueline N. Sanchez, MS,

Health Communication Specialists Will Yang, MA

Visual Information Specialist 


\section{Results}

During 2010-2018, the number of deaths among PWDH decreased by $7.5 \%$, from 16,742 during 2010 to 15,483 during 2018; the rate of death decreased by $36.6 \%$ overall (Figure 1 ). The rate of HIV-related deaths decreased $48.4 \%$ from 9.1 per 1,000 PWDH during 2010 to 4.7 per 1,000 PWDH during 2017, whereas the rate of non-HIV-related deaths decreased $8.6 \%$ from 9.3 in 2010 to 8.5 in 2017 (Figure 1). The rate of HIV-related deaths during 2010-2017 decreased in all regions and for all gender, age, race/ethnicity, and transmission category groups. (Supplementary Table 1, https://stacks.cdc. gov/view/cdc/96933). The absolute rate difference disparity measure for HIV-related deaths between Hispanic/Latino persons and White persons decreased to zero (3.9 per 1,000 PWDH in both populations) in 2017. During 2010-2017, the absolute rate difference disparity measure between Black/ African American (Black) persons and White persons decreased by $66.0 \%$, and between persons of multiple races and White persons decreased $36.7 \%$. The relative rate ratio disparity measure between Black persons and White persons decreased 23.2\%, between Hispanic/Latino persons and White persons decreased $17.7 \%$, but between persons of multiple races and White persons increased 2.3\%.

Rates of HIV-related deaths during 2017 were higher among females (5.4 per 1,000 PWDH) than males (4.5) and transgender females (females assigned male sex at birth) (4.3), and highest among persons of multiple races (7.0) and Black persons (5.6), followed by White persons (3.9) and Hispanic/ Latino persons (3.9) (Table 1). The rates of HIV-related deaths increased with age, from 1.6 among PWDH aged 13-24 years to 8.4 among persons aged $\geq 55$ years. However, the proportion of deaths that were HIV-related decreased with increasing age from 48.6\% among PWDH aged 13-24 years with a known cause of death to $30.0 \%$ among PWDH aged $\geq 55$ years with a known cause of death because the rate of non-HIV-related death increased with age more than the rate of HIV-related death. Among males, the rate of HIV-related death was lower among those whose infection was attributed to male-to-male sexual contact (3.9) than among those whose infection was attributed to other transmission categories; among females, the rate was lower among those with infection attributed to heterosexual contact (4.6) than among those in other transmission categories. The rate of HIV-related deaths was highest in the South (6.0) and lowest in the Northeast (3.2).

In all areas with complete cause-of-death reporting and with stable rates, HIV-related deaths were lower during 2017 than in 2010 (Supplementary Table 2, https://stacks.cdc.gov/ view/cdc/96934). Rates of HIV-related deaths during 2017 varied by jurisdiction; rates were highest in Mississippi (10.3 per 1,000 PWDH), Puerto Rico (9.2), and South Carolina
FIGURE 1. Age-adjusted rates* of total deaths, ${ }^{\dagger}$ human immunodeficiency virus (HIV)-related deaths, ${ }^{\S}$ and non-HIV-related deaths among persons aged $\geq 13$ years with diagnosed HIV infection - United States, 2010-2018

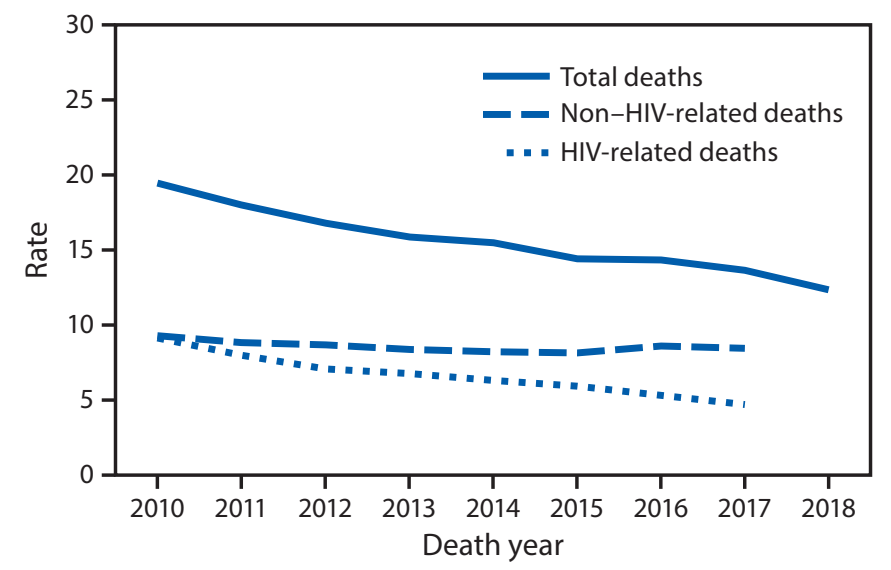

* Rates per 1,000 persons with diagnosed HIV infection. Rates age-adjusted using the U.S. 2000 standard population.

† Deaths among persons with diagnosed HIV infection regardless of cause of death $(\mathrm{n}=16,742$ in 2010; $\mathrm{n}=15,483$ in 2018).

$\S$ HIV-related deaths include deaths with an underlying cause with an International Classification of Diseases, Tenth Revision, code of B20-B24, O98.7, or R75. Non-HIV-related deaths include all other deaths with a known underlying cause.

๑ Deaths by cause available through 2017 because of reporting delays.

(8.0), and lowest in New York (3.0), Massachusetts (3.1) and Delaware (3.2) (Figure 2). During 2017, rates of HIV-related deaths by race/ethnicity varied by jurisdiction (Table 2) (Supplementary Table 3, https://stacks.cdc.gov/view/ cdc/96934). Rates of HIV-related death were highest among White persons in South Carolina (10.1), Oklahoma (7.5), and Arkansas (6.5); highest among Black persons in Mississippi (11.5), Louisiana (8.8), South Carolina (8.2), and Nevada (8.2); and highest among Hispanic/Latino persons in Puerto Rico (9.2), Texas (6.5), and Arizona (6.2).

\section{Discussion}

By 2018, the rate of death among PWDH in the United States had decreased by $36.6 \%$ from what it was in 2010 , surpassing the 2020 national target of $\geq 33 \%$ (7). This decrease, which was primarily attributable to reductions in HIV-related deaths, likely reflects the increase during 2010-2018 in the proportion of persons who knew their serostatus from $82.2 \%$ to $86.2 \%$ and the implementation of updated treatment guidelines resulting in increased viral suppression among PWDH from $46.0 \%$ to $64.7 \%(6,8)$. Absolute and relative differences in HIV-related deaths among Black persons and Hispanic/ Latino persons, compared with those among White persons, also decreased during 2010-2017. This reduction likely reflects a greater relative improvement during 2012-2017 in the time from diagnosis to viral suppression among Black persons, 
TABLE 1. Total deaths, human immunodeficiency virus (HIV)-related deaths, and non-HIV-related deaths among persons aged $\geq 13$ years with diagnosed HIV infection, by selected characteristics — United States, 2017

\begin{tabular}{|c|c|c|c|c|c|c|c|c|}
\hline \multirow[b]{2}{*}{ Characteristic } & \multicolumn{2}{|r|}{ Total } & \multicolumn{4}{|c|}{ HIV-related* } & \multicolumn{2}{|c|}{ Non-HIV-related* } \\
\hline & No. & $\begin{array}{l}\text { Age-adjusted rate } \\
\text { per } 1,000 \mathrm{PWDH}^{\dagger}\end{array}$ & No. & $\begin{array}{l}\% \text { of deaths } \\
\text { related to HIV }\end{array}$ & $\begin{array}{l}\% \text { of deaths with } \\
\text { known cause } \\
\text { related to HIV }\end{array}$ & $\begin{array}{l}\text { Age-adjusted rate } \\
\text { per } 1,000 \mathrm{PWDH}^{\dagger}\end{array}$ & No. & $\begin{array}{l}\text { Age-adjusted rate } \\
\text { per } 1,000 \mathrm{PWDH}^{\dagger}\end{array}$ \\
\hline \multicolumn{9}{|l|}{ Gender } \\
\hline Male & 12,256 & 13.5 & 4,033 & 32.9 & 34.1 & 4.5 & 7,779 & 8.5 \\
\hline Female & 3,994 & 14.1 & 1,466 & 36.7 & 37.7 & 5.4 & 2,425 & 8.3 \\
\hline Transgender male-to-female ${ }^{\S}$ & 103 & 14.8 & 33 & 32.0 & 32.4 & 4.3 & 69 & 10.2 \\
\hline Transgender female-to-male & 4 & 18.0 & 2 & 50.0 & 50.0 & 10.7 & 2 & 7.3 \\
\hline Additional gender identity" & 1 & 13.8 & 0 & 0.0 & 0.0 & 0.0 & 1 & 13.8 \\
\hline \multicolumn{9}{|l|}{ Age at death, yrs } \\
\hline $13-24$ & 151 & 3.5 & 70 & 46.4 & 48.6 & 1.6 & 74 & 1.7 \\
\hline $25-34$ & 1,048 & 6.5 & 492 & 46.9 & 48.8 & 3.0 & 516 & 3.2 \\
\hline $35-44$ & 1,838 & 9.4 & 826 & 44.9 & 46.4 & 4.2 & 954 & 4.9 \\
\hline $45-54$ & 4,470 & 14.4 & 1,584 & 35.4 & 36.6 & 5.1 & 2,740 & 8.9 \\
\hline$\geq 55$ & 8,851 & 28.9 & 2,562 & 28.9 & 30.0 & 8.4 & 5,992 & 19.5 \\
\hline \multicolumn{9}{|l|}{ Race/Ethnicity } \\
\hline $\begin{array}{l}\text { American Indian/ } \\
\text { Alaska Native }\end{array}$ & 44 & 13.5 & 8 & 18.2 & 21.1 & 3.3 & 30 & 8.3 \\
\hline Asian** & 88 & 6.3 & 27 & 30.7 & 39.1 & 1.5 & 42 & 3.1 \\
\hline Black/African American & 7,197 & 15.1 & 2,620 & 36.4 & 37.3 & 5.6 & 4,412 & 9.2 \\
\hline Hispanic/Latino ${ }^{+\dagger}$ & 2,694 & 11.1 & 955 & 35.4 & 37.2 & 3.9 & 1,609 & 6.6 \\
\hline $\begin{array}{l}\text { Native Hawaiian/ } \\
\text { Other Pacific Islander** }\end{array}$ & 9 & 15.0 & 0 & 0.0 & 0.0 & 0.0 & 2 & 2.5 \\
\hline White & 5,255 & 13.3 & 1,546 & 29.4 & 30.5 & 3.9 & 3,520 & 8.9 \\
\hline Multiple races & 1,069 & 19.5 & 378 & 35.4 & 36.5 & 7.0 & 659 & 12.0 \\
\hline \multicolumn{9}{|l|}{ Transmission category $\S^{\S}$} \\
\hline \multicolumn{9}{|l|}{ Male adult or adolescent ${ }^{\text {ๆๆ }}$} \\
\hline Male-to-male sexual contact & 7,010 & 11.4 & 2,408 & 34.4 & 35.6 & 3.9 & 4,351 & 7.1 \\
\hline Injection drug use & 2,168 & 22.7 & 590 & 27.2 & 28.1 & 6.2 & 1,506 & 15.8 \\
\hline $\begin{array}{l}\text { Male-to-male sexual contact } \\
\text { and injection drug use }\end{array}$ & 1,373 & 19.1 & 444 & 32.4 & 33.3 & 6.1 & 889 & 12.6 \\
\hline Heterosexual contact*** & 1,705 & 16.1 & 579 & 34.0 & 35.6 & 5.8 & 1,046 & 9.5 \\
\hline Othertt+ & 104 & 19.0 & 44 & 42.4 & 43.4 & 6.6 & 57 & 11.9 \\
\hline Subtotal & 12,360 & 13.5 & 4,066 & 32.9 & 34.1 & 4.5 & 7,849 & 8.5 \\
\hline \multicolumn{9}{|l|}{ Female adult or adolescent } \\
\hline Injection drug use & 1,373 & 21.6 & 454 & 33.0 & 33.7 & 7.7 & 893 & 13.1 \\
\hline Heterosexual contact ${ }^{* * *}$ & 2,553 & 12.0 & 974 & 38.2 & 39.3 & 4.6 & 1,506 & 7.1 \\
\hline Othert+† & 72 & 16.2 & 40 & 56.2 & 59.6 & 7.0 & 27 & 8.9 \\
\hline Subtotal & 3,998 & 14.1 & 1,468 & 36.7 & 37.7 & 5.4 & 2,427 & 8.3 \\
\hline \multicolumn{9}{|c|}{ U.S. Census region of residence at time of death } \\
\hline Midwest & 1,901 & 14.1 & 602 & 31.7 & 32.3 & 4.4 & 1,263 & 9.4 \\
\hline Northeast & 3,689 & 12.0 & 941 & 25.5 & 26.8 & 3.2 & 2,576 & 8.2 \\
\hline South & 8,040 & 15.5 & 3,092 & 38.5 & 39.1 & 6.0 & 4,822 & 9.2 \\
\hline West & 2,728 & 11.4 & 899 & 33.0 & 35.8 & 3.9 & 1,615 & 6.6 \\
\hline Total & 16,358 & 13.6 & 5,534 & 33.8 & 35.0 & 4.7 & 10,276 & 8.5 \\
\hline
\end{tabular}

Abbreviation: PWDH = persons with diagnosed HIV infection.

* HIV-related deaths include deaths with an underlying cause with an International Classification of Diseases, Tenth Revision code of B20-B24, O98.7, or R75. Non-HIVrelated deaths include all other deaths with a known underlying cause. Deaths with an unknown underlying cause are excluded.

${ }^{\dagger}$ PWDH includes persons living with HIV infection at the end of the calendar year plus the number of diagnoses of HIV infection during the current calendar year. Rates age-adjusted using the U.S. 2000 standard population. Rates presented by age at time of death are not age-adjusted. Rates and percentage change calculated on the basis of $<12$ deaths are considered unstable and should be interpreted with caution.

$\S$ "Transgender male-to-female" includes persons who were assigned "male" sex at birth but have ever identified as "female." "Transgender female-to-male" includes persons who were assigned "female" sex at birth but have ever identified as "male."

" Additional gender identity examples include "bigender," "gender queer," and "two-spirit."

** Data by cause of death should be interpreted with caution because $<85 \%$ of reported deaths were reported with a known underlying cause of death.

${ }^{+\dagger}$ Hispanic/Latino persons can be of any race.

$\S \S$ Data have been statistically adjusted to account for missing transmission category; therefore, values might not sum to column subtotals and total.

กๆ Data presented are based on sex at birth and include transgender persons.

*** Heterosexual contact with a person known to have, or to be at high risk for, HIV infection.

${ }^{+\dagger+}$ Includes hemophilia, blood transfusion, perinatal, and risk factor not reported or not identified. 
TABLE 2. Total deaths and human immunodeficiency virus (HIV)-related deaths among persons aged $\geq 13$ years with diagnosed HIV infection, by area of residence at time of death, and selected race/ethnicity categories — United States and Puerto Rico, 2017

\begin{tabular}{|c|c|c|c|c|c|c|c|c|c|c|c|c|c|c|c|}
\hline \multirow[b]{3}{*}{$\begin{array}{l}\text { Area of } \\
\text { residence }\end{array}$} & \multirow{2}{*}{\multicolumn{3}{|c|}{$\begin{array}{c}\text { All races/ethnicities } \\
\text { Total } \\
\end{array}$}} & \multicolumn{4}{|c|}{ Black/African American } & \multicolumn{4}{|c|}{ Hispanic/Latino } & \multicolumn{4}{|c|}{ White } \\
\hline & & & & \multicolumn{2}{|c|}{ Total } & \multicolumn{2}{|c|}{ HIV-related $^{\dagger}$} & \multicolumn{2}{|r|}{ Total } & \multicolumn{2}{|c|}{ HIV-related $^{\dagger}$} & \multicolumn{2}{|r|}{ Total } & \multicolumn{2}{|c|}{ HIV-related $^{\dagger}$} \\
\hline & No. & $\begin{array}{c}\text { Age- } \\
\text { adjusted } \\
\text { rate per } \\
1,000 \\
\text { PWDH }^{\S}\end{array}$ & $\begin{array}{c}\% \text { of } \\
\text { deaths } \\
\text { with a } \\
\text { known } \\
\text { cause }\end{array}$ & No. & $\begin{array}{c}\text { Age- } \\
\text { adjusted } \\
\text { rate per } \\
1,000 \\
\text { PWDH }^{\S}\end{array}$ & No. & $\begin{array}{c}\text { Age- } \\
\text { adjusted } \\
\text { rate per } \\
1,000 \\
\text { PWDH }^{\S}\end{array}$ & No. & $\begin{array}{c}\text { Age- } \\
\text { adjusted } \\
\text { rate per } \\
1,000 \\
\text { PWDH }^{\S}\end{array}$ & No. & $\begin{array}{c}\text { Age- } \\
\text { adjusted } \\
\text { rate per } \\
1,000 \\
\text { PWDH }^{\S}\end{array}$ & No. & $\begin{array}{c}\text { Age- } \\
\text { adjusted } \\
\text { rate per } \\
1,000 \\
\text { PWDH }^{\S}\end{array}$ & No. & $\begin{array}{c}\text { Age- } \\
\text { adjusted } \\
\text { rate per } \\
1,000 \\
\text { PWDH }^{\S}\end{array}$ \\
\hline Alabama & 257 & 17.8 & 98.4 & 150 & 17.5 & 60 & 6.5 & 5 & 11.4 & 2 & 5.2 & 83 & 19.5 & 29 & 6.1 \\
\hline Alaska & 7 & 8.5 & 100.0 & 0 & 0 & 0 & 0 & 1 & 11.8 & 0 & 0 & 5 & 14.0 & 1 & 1.6 \\
\hline Arizona & 247 & 13.5 & 99.6 & 29 & 14.9 & 10 & 6.1 & 56 & 12.3 & 26 & 6.2 & 145 & 14.7 & 48 & 4.6 \\
\hline Arkansas & 107 & 17.0 & 99.1 & 33 & 12.3 & 16 & 6.2 & 6 & 15.7 & 3 & 7.9 & 61 & 19.2 & 21 & 6.5 \\
\hline California & 1,717 & 11.0 & 89.7 & 327 & 12.1 & 118 & 4.4 & 509 & 10.2 & 195 & 3.9 & 724 & 10.4 & 193 & 2.8 \\
\hline Colorado & 132 & 8.4 & 100.0 & 13 & 5.9 & 6 & 2.8 & 33 & 12.2 & 12 & 4.9 & 77 & 7.2 & 33 & 3.1 \\
\hline Connecticut & 198 & 15.0 & 99.5 & 72 & 14.7 & 18 & 4.9 & 57 & 12.6 & 15 & 3.7 & 64 & 18.8 & 16 & 4.0 \\
\hline Delaware & 67 & 13.8 & 100.0 & 50 & 18.0 & 13 & 4.6 & 4 & 13.6 & 1 & 3.8 & 11 & 6.5 & 1 & 0.5 \\
\hline $\begin{array}{l}\text { District of } \\
\text { Columbia }\end{array}$ & 237 & 13.4 & 97.0 & 202 & 16.2 & 59 & 5.3 & 3 & 3.5 & 1 & 1.2 & 14 & 4.6 & 2 & 0.6 \\
\hline Florida & 2,122 & 15.6 & 98.4 & 1,049 & 17.5 & 424 & 7.2 & 329 & 11.0 & 124 & 4.1 & 683 & 16.2 & 199 & 4.7 \\
\hline Georgia & 823 & 14.5 & 97.7 & 559 & 15.3 & 244 & 6.5 & 43 & 12.5 & 18 & 4.2 & 158 & 12.0 & 38 & 2.9 \\
\hline Hawaiiף & 37 & 15.2 & 35.1 & 1 & 5.6 & 0 & 0 & 1 & 3.3 & 1 & 3.3 & 12 & 7.0 & 2 & 0.8 \\
\hline Idaho & 20 & 13.6 & 100.0 & 0 & 0 & 0 & 0 & 1 & 2.6 & 1 & 2.6 & 17 & 16.4 & 6 & 5.8 \\
\hline Illinois & 498 & 12.8 & 99.2 & 289 & 16.6 & 76 & 4.4 & 48 & 7.3 & 14 & 1.8 & 124 & 11.2 & 30 & 2.1 \\
\hline Indiana & 212 & 17.1 & 97.2 & 66 & 15.7 & 20 & 5.4 & 10 & 10.6 & 6 & 6.4 & 126 & 20.3 & 44 & 5.6 \\
\hline lowa & 44 & 13.1 & 100.0 & 11 & 16.9 & 6 & 9.7 & 3 & 7.0 & 1 & 2.3 & 26 & 11.3 & 10 & 4.4 \\
\hline Kansas & 47 & 14.4 & 97.9 & 11 & 16.0 & 7 & 11.1 & 6 & 10.8 & 2 & 2.1 & 29 & 14.1 & 12 & 5.1 \\
\hline Kentucky & 138 & 16.4 & 97.8 & 44 & 17.0 & 17 & 6.3 & 5 & 6.4 & 3 & 3.9 & 82 & 16.8 & 24 & 4.8 \\
\hline Louisiana & 411 & 18.5 & 98.8 & 276 & 19.0 & 125 & 8.8 & 7 & 5.8 & 1 & 0.7 & 115 & 19.8 & 34 & 6.3 \\
\hline Maine & 35 & 15.6 & 100.0 & 1 & 2.5 & 0 & 0 & 2 & 12.0 & 1 & 4.9 & 31 & 19.0 & 7 & 5.9 \\
\hline Maryland & 597 & 14.7 & 98.3 & 412 & 14.0 & 132 & 4.4 & 26 & 9.5 & 9 & 3.0 & 84 & 18.9 & 19 & 3.8 \\
\hline Massachusetts & 307 & 11.6 & 96.1 & 64 & 8.3 & 14 & 2.6 & 90 & 13.1 & 27 & 4.2 & 144 & 13.7 & 33 & 2.8 \\
\hline Michigan & 273 & 15.6 & 99.3 & 160 & 17.2 & 49 & 5.1 & 13 & 14.9 & 4 & 3.2 & 88 & 12.5 & 27 & 3.8 \\
\hline Minnesota & 91 & 9.3 & 98.9 & 27 & 8.3 & 15 & 4.0 & 6 & 6.9 & 3 & 2.1 & 52 & 10.2 & 19 & 4.2 \\
\hline Mississippi & 220 & 21.2 & 98.6 & 158 & 22.1 & 85 & 11.5 & 9 & 36.7 & 6 & 21.3 & 44 & 19.3 & 12 & 5.5 \\
\hline Missouri & 213 & 14.5 & 97.2 & 97 & 16.7 & 38 & 6.5 & 10 & 13.1 & 6 & 7.8 & 95 & 13.6 & 30 & 3.9 \\
\hline Montana & 15 & 20.9 & 100.0 & 0 & 0 & 0 & 0 & 4 & 93.8 & 2 & 34.1 & 8 & 11.4 & 1 & 1.6 \\
\hline Nebraska & 33 & 14.3 & 93.9 & 11 & 20.2 & 4 & 5.7 & 2 & 6.9 & 1 & 5.4 & 20 & 14.9 & 4 & 2.5 \\
\hline Nevada & 174 & 16.1 & 97.7 & 50 & 20.5 & 19 & 8.2 & 23 & 9.6 & 9 & 3.4 & 90 & 15.9 & 26 & 4.6 \\
\hline $\begin{array}{l}\text { New } \\
\text { Hampshire }\end{array}$ & 21 & 13.7 & 95.2 & 0 & 0 & 0 & 0 & 3 & 15.5 & 0 & 0 & 18 & 17.2 & 6 & 6.0 \\
\hline New Jersey & 646 & 13.8 & 98.6 & 324 & 15.1 & 105 & 5.2 & 141 & 12.1 & 43 & 3.9 & 118 & 11.9 & 25 & 3.0 \\
\hline New Mexico & 61 & 13.1 & 98.4 & 2 & 8.4 & 0 & 0 & 24 & 11.2 & 4 & 2.0 & 25 & 12.4 & 3 & 1.4 \\
\hline New York & 1,787 & 10.7 & 98.3 & 661 & 10.7 & 164 & 3.0 & 635 & 11.0 & 170 & 3.0 & 247 & 7.2 & 59 & 1.9 \\
\hline North Carolina & 547 & 15.1 & 98.9 & 338 & 15.3 & 137 & 6.1 & 19 & 7.9 & 8 & 3.5 & 152 & 15.1 & 48 & 4.6 \\
\hline North Dakota & 3 & 8.9 & 100.0 & 0 & 0 & 0 & 0 & 1 & 131.2 & 1 & 131.2 & 2 & 8.7 & 1 & 4.4 \\
\hline Ohio & 377 & 15.5 & 97.9 & 152 & 15.2 & 53 & 5.5 & 21 & 10.7 & 7 & 3.7 & 176 & 16.7 & 50 & 4.2 \\
\hline Oklahoma & 109 & 16.8 & 95.4 & 22 & 13.5 & 11 & 7.1 & 6 & 8.9 & 1 & 1.0 & 65 & 20.3 & 22 & 7.5 \\
\hline Oregon & 115 & 12.4 & 100.0 & 5 & 9.6 & 1 & 1.9 & 9 & 7.9 & 6 & 5.3 & 96 & 13.4 & 36 & 5.1 \\
\hline Pennsylvania? & 650 & 14.5 & 81.8 & 301 & 14.4 & 55 & 2.5 & 105 & 14.7 & 21 & 3.0 & 202 & 14.4 & 34 & 2.4 \\
\hline Puerto Rico & 401 & 19.1 & 99.0 & 0 & 0 & 0 & 0 & 400 & 19.1 & 181 & 9.2 & 1 & 21.9 & 0 & 0 \\
\hline Rhode Island & 33 & 9.6 & 97.0 & 7 & 8.6 & 3 & 3.5 & 6 & 8.6 & 2 & 3.2 & 17 & 9.5 & 4 & 2.2 \\
\hline $\begin{array}{l}\text { South } \\
\text { Carolina }\end{array}$ & 337 & 17.2 & 97.9 & 236 & 17.7 & 110 & 8.2 & 5 & 6.4 & 2 & 2.3 & 83 & 19.3 & 34 & 10.1 \\
\hline South Dakota? & 9 & 12.4 & 33.3 & 2 & 9.1 & 1 & 3.9 & 0 & 0 & 0 & 0 & 4 & 10.0 & 0 & 0 \\
\hline Tennessee & 332 & 18.2 & 99.4 & 189 & 19.9 & 81 & 7.7 & 6 & 9.2 & 2 & 3.2 & 128 & 17.3 & 48 & 5.8 \\
\hline Texas & 1,413 & 15.1 & 98.9 & 557 & 17.3 & 245 & 7.4 & 359 & 12.8 & 182 & 6.5 & 409 & 14.6 & 157 & 5.8 \\
\hline Utah & 22 & 8.4 & 95.5 & 1 & 4.2 & 1 & 4.2 & 3 & 9.8 & 0 & 0 & 16 & 7.0 & 8 & 3.4 \\
\hline Vermont & 12 & 11.4 & 100.0 & 0 & 0 & 0 & 0 & 1 & 7.5 & 0 & 0 & 10 & 12.2 & 3 & 3.2 \\
\hline Virginia & 292 & 10.5 & 98.3 & 180 & 11.8 & 60 & 4.1 & 9 & 3.3 & 6 & 2.1 & 79 & 9.0 & 27 & 3.2 \\
\hline Washington & 175 & 10.4 & 97.1 & 24 & 10.8 & 9 & 4.2 & 19 & 9.3 & 6 & 2.8 & 112 & 10.8 & 35 & 3.8 \\
\hline West Virginia & 31 & 13.8 & 100.0 & 4 & 7.5 & 1 & 2.1 & 1 & 11.9 & 0 & 0 & 24 & 15.8 & 6 & 4.6 \\
\hline Wisconsin & 101 & 12.7 & 100.0 & 30 & 11.7 & 8 & 3.3 & 9 & 9.0 & 0 & 0 & 57 & 13.8 & 18 & 4.2 \\
\hline Wyoming" & 6 & 27.2 & 66.7 & 0 & 0 & 0 & 0 & 0 & 0 & 0 & 0 & 3 & 9.5 & 1 & 3.5 \\
\hline
\end{tabular}

See table footnotes on the next page. 
Morbidity and Mortality Weekly Report

TABLE 2. (Continued) Total deaths and human immunodeficiency virus (HIV)-related deaths among persons aged $\geq 13$ years with diagnosed HIV infection, by area of residence at time of death, and selected race/ethnicity categories — United States and Puerto Rico, ${ }^{*} 2017$

Abbreviation: PWDH = persons with diagnosed HIV infection.

* Other U.S. dependent areas are excluded because they do not report underlying cause of death information.

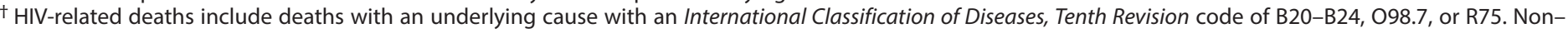
HIV-related deaths include all other deaths with a known underlying cause. Deaths with an unknown underlying cause are excluded.

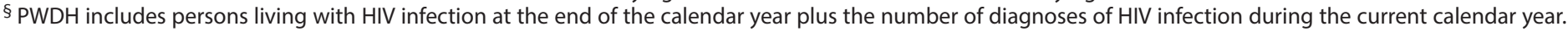

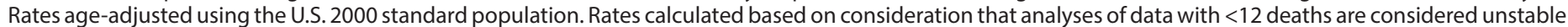
and should be interpreted with caution.

I Proportion of deaths with a known underlying cause of death is $<85 \%$.

compared with White persons (9), and reduced disparities during 2010-2016 in viral suppression among Black persons and Hispanic/Latino persons, compared with White persons (10). These findings highlight how successes in identifying HIV infections, initiating treatment, and achieving viral suppression among PWDH improve health outcomes.

Despite success in reducing rates of HIV-related deaths among PWDH, differences still exist by gender, race/ethnicity, age, transmission category, and region. Variation in timely diagnosis and treatment initiation, along with ongoing treatment, likely contributes to differences in HIV-related deaths. During 2015, delays in HIV diagnosis were longer among non-White racial/ethnic groups and males with HIV infection attributed to heterosexual contact (11). Timely initiation of treatment, as measured by the proportion of persons with suppressed viral loads $\leq 6$ months after diagnosis, and receipt of ongoing, recommended treatment, as measured by the proportion of PWDH with a suppressed viral load, varied during 2017 by gender, age, race/ethnicity, transmission category, and region $(8,12)$; populations with higher rates of HIV-related deaths were less likely to have evidence of timely initiation of treatment and ongoing treatment as demonstrated through lower proportions of viral suppression in the population.

Prevalence of HIV infection and the number of HIV-related deaths were greatest by race/ethnicity among Black persons and by U.S. region in the South (4). Rates of HIV-related deaths were also high among these two populations. Higher levels of poverty, unemployment, and persons uninsured, challenges associated with accessing care, and HIV-related stigma likely affect timely diagnosis and access to treatment and contribute to higher rates of HIV-related deaths $(13,14)$. Expanded efforts to address these and other structural barriers are critical to improving health outcomes, including reducing differences in HIV-related death rates, especially among Black persons and persons in the South.

Although rates of HIV-related deaths were lower among younger PWDH, the proportion of HIV-related deaths among younger PWDH (ages 13-44 years) was higher than that among older PWDH; this is concerning because HIV-related deaths are preventable. Higher proportions of undiagnosed HIV infections and lower levels of viral suppression are more common among younger persons $(8,15)$. Additional efforts are needed to ensure younger persons are aware of their infection and able to access and adhere to recommended, ongoing HIV treatment to improve health outcomes.

CDC supports numerous activities for identifying HIV infections: initiating treatment as quickly as possible and ensuring ongoing treatment; addressing social barriers to HIV prevention and treatment efforts; and expanding opportunities for persons to test for HIV infection and receive the results on their own (i.e., self-testing), which allows persons who might not otherwise take a test to learn their HIV status (16). CDC's Integrated HIV Surveillance and Prevention Programs for Health Departments, initiated in 2018, includes critical activities to enable state and local health departments to improve identification of HIV infections and increase viral suppression among PWDH (17). CDC's national campaign, Let's Stop HIV Together, supports efforts to end HIV stigma and promote HIV testing, prevention, and treatment (18). Ending the HIV Epidemic: A Plan for America is an initiative for reducing HIV infections in the United States by $\geq 90 \%$ by 2030 ; it focuses on strategies regarding diagnosis, treatment, prevention, and response to HIV infection in communities most affected by HIV (19). In addition to decreasing the risk for ongoing HIV transmission, prompt diagnosis and improving timely and continuing access to HIV treatment should also improve health outcomes for PWDH and prevent HIV-related deaths.

The findings in this report are subject to at least two limitations. First, cause-of-death information on death certificates is typically completed by funeral directors, attending physicians, medical examiners, or coroners (3). HIV-related deaths might be underreported because of lack of knowledge about the correct documentation needed or reluctance to include HIV on the death certificate because of possible stigma (5). An assessment of Florida's HIV surveillance data for 2000-2011 indicated that HIV-related deaths were underestimated in the surveillance system by approximately $9 \%(20)$. Second, the proportion of deaths with a known cause was $<100 \%$. Overall, the proportion of deaths with a known cause was high for the United States (94.6\% in 2010 and 96.7\% in 2017); however, the proportion of deaths with a known cause was lower for 
FIGURE 2. Age-adjusted rates* of human immunodeficiency virus (HIV)-related deaths among persons aged $\geq 13$ years with diagnosed HIV infection, by area of residence at time of death - United States and Puerto Rico, 2017

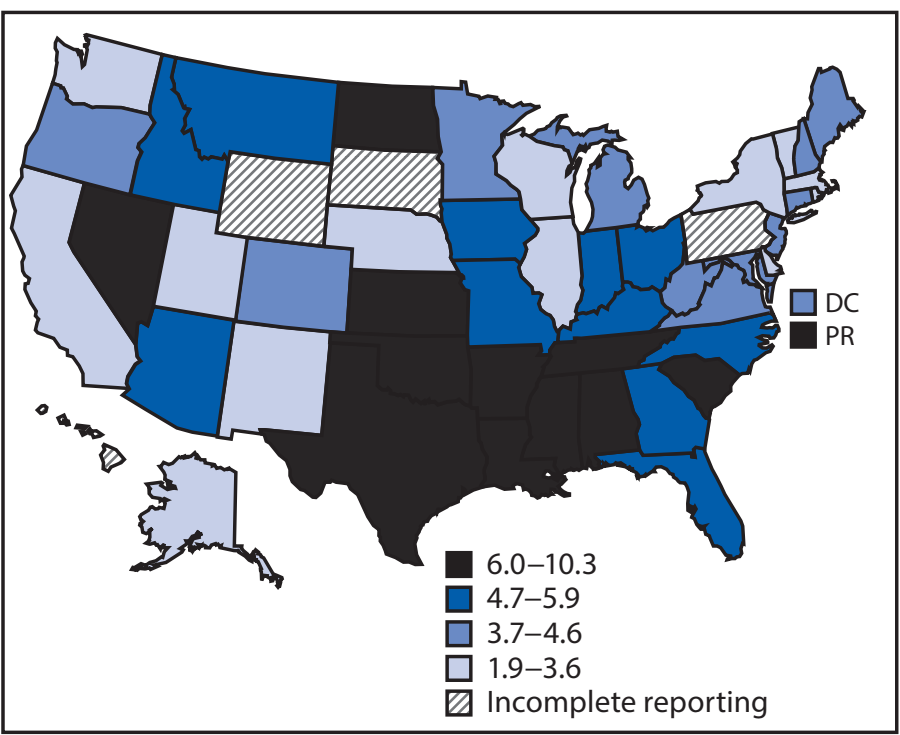

Abbreviations: $\mathrm{DC}=$ District of Columbia; $\mathrm{PR}=$ Puerto Rico.

* Rates per 1,000 persons with diagnosed HIV infection. Rates age-adjusted using the U.S. 2000 standard population. HIV-related deaths include deaths with an underlying cause with an International Classification of Diseases, Tenth Revision code of B20-B24, 098.7, or R75. Other U.S. dependent areas are excluded because they do not report underlying cause of death information. Jurisdictions with striped shading are those with $<85 \%$ of deaths in 2017 with a known underlying cause of death. Rates from Alaska, Idaho, Maine, Montana, Nebraska, New Hampshire, New Mexico, North Dakota, Rhode Island, Utah, Vermont, and West Virginia are calculated based on $<12$ deaths and should be interpreted with caution.

certain demographic groups (e.g., Asian persons) and for certain jurisdictions (e.g., Hawaii during 2017).

Deaths among persons with HIV have decreased, and by 2018 had surpassed the 2020 national target, primarily because of a reduction in HIV-related deaths. Deaths caused by HIV infection have likely decreased because of improvements in diagnosing infections and in treatment and medical care. However, differences in HIV-related death rates still exist for multiple populations. Diagnosing HIV infection early, treating it promptly, and maintaining access to high-quality care and treatment over a lifetime can improve life expectancy and reduce differences in rates of deaths across all populations.

\section{Acknowledgment}

Xueyuan (Bill) Dong, ICF Corporation, Atlanta, Georgia. Corresponding author: Karin A. Bosh, hxx8@cdc.gov, 404-639-3615.

${ }^{1}$ Division of HIV/AIDS Prevention, National Center for HIV/AIDS, Viral Hepatitis, STD, and TB Prevention, CDC.

All authors have completed and submitted the International Committee of Medical Journal Editors form for disclosure of potential conflicts of interest. No potential conflicts of interest were disclosed.

\section{Summary}

What is already known about this topic?

HIV remains among the 10 leading causes of death among certain populations, although deaths attributable to HIV infection are preventable.

What is added by this report?

Deaths among persons with diagnosed HIV (PWDH) decreased, primarily because of decreases in HIV-related deaths. The age-adjusted rate per 1,000 PWDH of HIV-related deaths decreased $48 \%$ and non-HIV-related deaths decreased $9 \%$ during 2010-2017. Differences in HIV-related deaths persist for certain populations.

What are the implications for public health practice?

Continued efforts in diagnosing HIV early, promptly initiating treatment, and maintaining access to high-quality care and treatment are necessary for continuing progress in reducing deaths and eliminating differences across populations.

\section{References}

1. Samji H, Cescon A, Hogg RS, et al.; North American AIDS Cohort Collaboration on Research and Design (NA-ACCORD) of IeDEA. Closing the gap: increases in life expectancy among treated HIV-positive individuals in the United States and Canada. PLoS One 2013;8:e81355. PMID:24367482 https://doi.org/10.1371/journal.pone.0081355

2. Marcus JL, Leyden W, Anderson AN, et al. Increased overall life expectancy but not comorbidity-free years for people with HIV [abstract 151]. Presented at the 2020 Conference on Retroviruses and Opportunistic Infections; March 8-11, 2020; Boston, Massachusetts. https://www.croiconference.org/abstract/increased-overall-lifeexpectancy-but-not-comorbidity-free-years-for-people-with-hiv/

3. Heron M. Deaths: leading causes for 2017. National Vital Statistics report vol. 68, no. 6. Atlanta, GA: US Department of Health and Human Services, CDC; 2019. https://www.cdc.gov/nchs/data/nvsr/ nvsr68/nvsr68_06-508.pdf

4. CDC. Diagnoses of HIV infection in the United States and dependent areas, 2018. HIV surveillance report 2018 (updated), vol. 31. Atlanta, GA: US Department of Health and Human Services, CDC; 2020. https:// www.cdc.gov/hiv/library/reports/hiv-surveillance/vol-31/index.html

5. Adih WK, Selik RM, Hall HI, Babu AS, Song R. Associations and trends in cause-specific rates of death among persons reported with HIV infection, 23 U.S. jurisdictions, through 2011. Open AIDS J 2016;10:144-57. PMID:27708746 https://doi.org/10.2174/1874613601610010144

6. Panel on Antiretroviral Guidelines for Adults and Adolescents. Guidelines for the use of antiretroviral agents in HIV-1 infected adults and adolescents. Washington, DC: US Department of Health and Human Services; 2012. https://aidsinfo.nih.gov/contentfiles/ AdultandAdolescentGL003093.pdf

7. White House Office on National AIDS Policy. National HIV/AIDS strategy for the United States: updated to 2020. Washington, DC: Office of National AIDS Policy; 2015. https://files.hiv.gov/s3fs-public/ nhas-update.pdf

8. CDC. Monitoring selected national HIV prevention and care objectives by using HIV surveillance data-United States and 6 dependent areas, 2018. HIV surveillance supplemental report, vol. 25, no. 2. Atlanta, GA: US Department of Health and Human Services, CDC; 2020. https://www.cdc.gov/hiv/pdf/library/reports/surveillance/cdc-hivsurveillance-supplemental-report-vol-25-2.pdf 
9. Crepaz N, Song R, Lyss S, Hall HI. Trends in time from HIV diagnosis to first viral suppression following revised U.S. HIV treatment guidelines, 2012-2017. J Acquir Immune Defic Syndr 2020;85:46-50. PMID:32379083 https://doi.org/10.1097/QAI.0000000000002398

10. Mandsager P, Marier A, Cohen S, Fanning M, Hauck H, Cheever LW. Reducing HIV-related health disparities in the Health Resources and Services Administration's Ryan White HIV/AIDS Program. Am J Public Health 2018;108(Suppl4):S246-50 10. PMID:30383416 https://doi. org/10.2105/AJPH.2018.304689

11. Dailey AF, Hoots BE, Hall HI, et al. Vital signs: human immunodeficiency virus testing and diagnosis delays-United States. MMWR Morb Mortal Wkly Rep 2017;66:1300-6. PMID:29190267 https://doi. org/10.15585/mmwr.mm6647e1

12. Harris NS, Johnson AS, Huang YA, et al. Vital signs: status of human immunodeficiency virus testing, viral suppression, and HIV preexposure prophylaxis_-United States, 2013-2018. MMWR Morb Mortal Wkly Rep 2019;68:1117-23. PMID:31805031 https://doi.org/10.15585/ mmwr.mm6848e1

13. CDC. HIV by race/ethnicity. Atlanta, GA: US Department of Health and Human Services, CDC; 2019. https://www.cdc.gov/hiv/group/ racialethnicl

14. CDC. HIV in the southern United States. Atlanta, GA: US Department of Health and Human Services, CDC; 2019. https://www.cdc.gov/hiv/ pdf/policies/cdc-hiv-in-the-south-issue-brief.pdf
15. CDC. Estimated HIV incidence and prevalence in the United States, 2014-2018. HIV surveillance supplemental report, vol. 25, no. 1. Atlanta, GA: US Department of Health and Human Services, CDC; 2020. https://www.cdc.gov/hiv/pdf/library/reports/surveillance/cdc-hivsurveillance-supplemental-report-vol-25-1.pdf

16. CDC. HIV self-testing. Atlanta, GA: US Department of Health and Human Services, CDC; 2020. https://www.cdc.gov/hiv/testing/selftesting.html

17. CDC. Funding opportunity announcement (FOA) PS18-1802: integrated human immunodeficiency virus (HIV) surveillance and prevention programs for health departments. Atlanta, GA: US Department of Health and Human Services, CDC; 2017. https://www. cdc.gov/hiv/funding/announcements/ps18-1802/

18. CDC. Let's stop HIV together. Atlanta, GA: US Department of Health and Human Services, CDC; 2020. https://www.cdc.gov/stophivtogether/

19. CDC. Ending the HIV epidemic: a plan for America. Atlanta, GA: US Department of Health and Human Services, CDC; 2020. https://www. cdc.gov/endhiv/index.html

20. Trepka MJ, Sheehan DM, Fennie KP, Niyonsenga T, Lieb S, Maddox LM. Completeness of HIV reporting on death certificates for Floridians reported with HIV infection, 2000-2011. AIDS Care 2016;28:98-103. PMID:26273965 https://doi.org/10.1080/09540121.2015.1069786 